Provided for non-commercial research and educational use. Not for reproduction, distribution or commercial use.
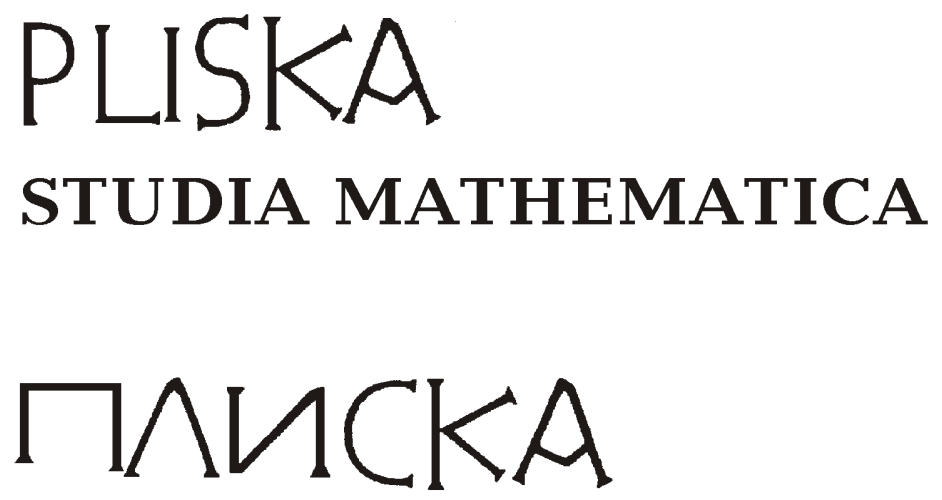

МАТЕМАТИЧЕСКИ СТУАИИ

The attached copy is furnished for non-commercial research and education use only. Authors are permitted to post this version of the article to their personal websites or institutional repositories and to share with other researchers in the form of electronic reprints.

Other uses, including reproduction and distribution, or selling or licensing copies, or posting to third party websites are prohibited.

For further information on

Pliska Studia Mathematica

visit the website of the journal http://www.math.bas.bg/ pliska/

or contact: Editorial Office

Pliska Studia Mathematica

Institute of Mathematics and Informatics

Bulgarian Academy of Sciences

Telephone: (+359-2)9792818, FAX:(+359-2)971-36-49

e-mail: pliska@math.bas.bg 


\title{
CRITICAL MARKOV BRANCHING PROCESSES WITH NON-HOMOGENEOUS POISSON IMMIGRATION
}

\author{
Kosto V. Mitov, Nikolay M. Yanev
}

\begin{abstract}
The paper deals with critical Markov branching processes with infinite offspring variance allowing an immigration component at the jump points of a time inhomogeneous Poisson process. The asymptotic formulas for the probability for non extinction are obtained depending on the rate of change of the intensity of the Poisson process. Proper limiting distributions are proved under the appropriate normalization.
\end{abstract}

\section{Introduction}

The first model of branching processes with immigration was introduced and investigated by Sevastyanov [11]. He considered the continuous-time Markov branching process when the moments of immigration form a homogeneous Poisson process. The Sevastyanov immigration model was considered in the discrete time case, for Galton-Watson processes, by Heathcote [4]. The discrete time model was further developed by many authors (see for example the book of Athreya and Ney [1] and references therein). Especially Foster [3] and Pakes [8] considered a discrete time branching process with immigration only in the state zero.

It is well known that the behavior of critical branching processes without immigration essentially defers in the cases of finite and infinite offspring variance. The famous exponential limit law in the case of finite variance was proved by Yaglom [16] for the processes with discrete and continuous time Markov case. The

2010 Mathematics Subject Classification: 60J80

Key words: Branching processes, Immigration, Poisson process, Limit theorems; 
limiting behavior of critical Markov branching processes with infinite offspring variance were studied later by Zolotarev [18] and Slack [13].

The critical Markov processes with homogeneous Poisson immigration also have different limiting behavior in cases of finite or infinite variance. The Gamma distribution proved by Sevastyanov [11] differs from the limit laws proved by Vatutin [15].

In the recent papers of Hyrien et al. [5, 6] the authors study subcritical and supercritical Markov branching process with non-homogeneous Poisson immigration. The critical branching processes with finite variance allowing nonhomogeneous Poisson immigration have been studied by Mitov and Yanev [7] in much more general situation, namely for Sevastyanov branching model. The results obtained there can be easily transferred to the Markov processes with finite offspring variance and non-homogeneous Poisson immigration.

In the present paper we investigate the critical Markov branching processes with non-homogeneous Poisson immigration if the variance of the offspring of one particle is infinite. Instead of studying the process in the general settings we restrict us to the case when the probability generating functions have an explicit form (see Sevastyanov [10] or Sagitov and Lindo [12]).

\section{Markov branching processes}

Markov branching process can be described as follows (see for details Athreya and Ney [1]). The particles of a given type evolve in a broad-media. Each particle, independently of the others, lives random time $\tau$ with exponential distribution function $G(t)=\mathbf{P}\{\tau \leq t\}=1-e^{-t / \mu}, t \geq 0$, and at the end of its life the particle produces random number $\xi \geq 0$ of new particles of the same type. Each of them evolves in the same way. If we assume that this evolution started at time $t=0$ with one new particle, then the number of particles $Z(t)$ at every moment $t \geq 0$ forms a Markov branching process.

Denote by $h(s)=\mathbf{E}\left[s^{\xi}\right]$ the offspring p.g.f. and let $F(t ; s)=\mathbf{E}\left[s^{Z(t)}\right], \quad t \geq$ $0, s \in[0,1]$ be the p.g.f. of the process $Z(t), \quad t \geq 0$. It is well known that $F(t+\tau ; s)=F(t ; F(\tau ; s))$ for every $t, \tau \geq 0$. The p.g.f. $F(t ; s)$ satisfies the following non-linear integral equation

$$
F(t ; s)=s e^{-t / \mu}+\frac{1}{\mu} \int_{0}^{t} h(F(t-u, s)) e^{-u / \mu} d u
$$

with initial condition $F(0 ; s)=s$, and, under mild regularity conditions, it is the only solution of this equation in the class of p.g.f.

A Markov branching process is said to be subcritical, critical, or supercritical if $a=h^{\prime}(1)<1, \quad a=h^{\prime}(1)=1$, or $a=h^{\prime}(1)>1$, respectively. Further we will 
consider the critical case $(a=1)$ with infinite offspring variance. In this case we assume that as $s \uparrow 1$,

$$
h(s)=s+(1-s)^{\gamma+1} L\left(\frac{1}{1-s}\right), \quad \gamma \in(0,1]
$$

Under these conditions

$$
\bar{F}(t):=1-F(t ; 0) \sim t^{-1 / \gamma} L_{1}(t), \quad t \rightarrow \infty .
$$

The following limit theorem is due to Zolotarev [18]. (See also the recent paper of Pakes [9]).

Theorem 1. ([9]) If the condition (2.1) holds then

$$
\lim _{t \rightarrow \infty} \mathbf{P}\{\bar{F}(t) Z(t) \leq x \mid Z(t)>0\}=D_{\gamma}(x),
$$

where

$$
\hat{D}_{\gamma}(\lambda)=\int_{0}^{\infty} e^{-\lambda t} d D_{\gamma}(t)=1-\left(1+\lambda^{-\gamma}\right)^{-1 / \gamma}, \quad \lambda \geq 0
$$

\section{Processes with non-homogeneous Poisson immigration}

Let us suppose that along the Markov branching process $Z(t)$ there is a sequence of random vectors $\left(S_{k}, I_{k}\right), k=0,1,2, \ldots$, independent of $Z(t)$, where

$$
0=S_{0}<S_{1}<S_{2}<S_{3}<\ldots
$$

are the jump points of a non-homogeneous Poisson process $\nu(t)$ independent of $Z(t)$ and $I_{k}$ are i.i.d. non-negative integer valued random variables. Denote by $r(t)$ the intensity of $\nu(t), R(t)=\int_{0}^{t} r(u) d u$, and by $g(s)=\sum_{k=0}^{\infty} q_{k} s^{i}$ the p.g.f. of $I_{k}$.

Assume that at every jump-point $S_{n}$, a random number $I_{n}$ of new particles immigrate into the process $Z(t)$ and they participate in the evolution as the other particles. Let us denote the new process by $Y(t)$. It can be represent as follows

$$
Y(t)= \begin{cases}\sum_{k=1}^{\nu(t)} Z_{(k)}\left(t-S_{k}\right), & \nu(t)>0 \\ Y(t)=0, & \nu(t)=0\end{cases}
$$

where $Z_{(k)}(t)$ have the same branching mechanism as $Z(t)$, but they started with random number of ancestors at random time, i.e. $Z_{(k)}\left(t-S_{k}\right)$ starts with $I_{k}$ ancestors at the moment $S_{k}$.

Definition 1. The process $Y(t), t \geq 0$, is called Markov branching process with non-homogeneous Poisson immigration (MBPNPI). 
The probability generating function of the process $Y(t)$ has the following form (see Yakovlev and Yanev[17]),

$$
\Phi(t ; s):=\mathbf{E}\left[s^{Y(t)}\right]=\exp \left\{-\int_{0}^{t} r(t-u)(1-g(F(u ; s))) d u\right\}
$$

\section{Basic assumptions and notations}

In the next sections we will consider the process under the following assumptions:

- We assume that the offspring p.g.f. is $h(s)=\mathbf{E}\left[s^{\xi}\right]=s+(1-s)^{1+\gamma}$, $0<\gamma<1$, and $G(t)=1-e^{-t / \mu}, \quad t \geq 0$.

Then the probability generating function of the the process $Z(t), t \geq 0$ has the following explicit form

$$
F(t ; s)=1-\frac{1-s}{\left(1+c t(1-s)^{\gamma}\right)^{1 / \gamma}}, \quad t>0, \quad s \in[0,1) .
$$

where $c=\frac{\gamma}{(1+\gamma) \mu} \in(0, \infty)$.

This example is given in the Sevastyanov's book [10] (see also the recent paper of Sagitov and Lindo [12]).

The process is critical, that is the number of the offspring of one particle is a random variable with mean one and infinite variance.

For the intensity of the Poisson process we assume the following conditions:

$$
\begin{aligned}
& r(t)=\frac{\rho}{(t+1)^{\theta}}, t \geq 0, \\
& r(t) \uparrow \rho>0, \quad t \rightarrow \infty, \\
& r(t)=\rho t^{\theta}, t \geq 0,
\end{aligned}
$$

where $\rho$ and $\theta$ are positive constants.

- We assume also that only one particle immigrates at the every jump point of the Poisson process, that is $g(s)=s$.

Under these assumptions the probability generating function of the process $Y(t)$ has the following form (see (3.1)),

$$
\begin{aligned}
\Phi(t ; s) & =\mathbf{E}\left[s^{Y(t)}\right]=\exp \left\{-\int_{0}^{t} r(t-u) \bar{F}(u ; s) d u\right\} \\
& =\exp \left\{-\int_{0}^{t} \frac{(1-s) r(t-u)}{\left(1+c u(1-s)^{\gamma}\right)^{1 / \gamma}} d u\right\},
\end{aligned}
$$


where

$$
\bar{F}(t ; s)=1-F(t ; s)=\frac{(1-s)}{\left(1+c u(1-s)^{\gamma}\right)^{1 / \gamma}}
$$

\section{Probability for non extinction}

In this section we prove the asymptotic formulas for the probability for non extinction under the above basic assumptions. We will use the following notations

$$
\begin{aligned}
& \bar{F}(t)=\bar{F}(t ; 0)=1-F(t ; 0)=\frac{1}{(1+c t)^{1 / \gamma}} \\
& Q(t)=\int_{0}^{t} \bar{F}(u) d u=\frac{\gamma}{c(1-\gamma)}\left(1-(1+c t)^{1-1 / \gamma}\right) \\
& Q=Q(0)=\int_{0}^{\infty} \bar{F}(t) d t=\frac{\gamma}{c(1-\gamma)} \in(0, \infty)
\end{aligned}
$$

Setting $s=0$ in (4.5) we have for the probability for non extinction

$$
\mathbf{P}\{Y(t)>0\}=1-\Phi(t ; 0)=1-\exp \left\{-\int_{0}^{t} r(t-u) \bar{F}(u) d u\right\} .
$$

Theorem 2. Assume that (4.1) and (4.2) holds.

(a) If $\theta>1$, then

$$
\mathbf{P}\{Y(t)>0\} \sim Q r(t)+R \bar{F}(t)=\frac{\gamma}{c(1-\gamma)} r(t)+\frac{\rho}{\theta-1}(1+c t)^{-1 / \gamma}, t \rightarrow \infty,
$$

where $R=\int_{0}^{\infty} r(t) d t=\frac{\rho}{\theta-1} \in(0, \infty)$.

(b) If $\theta \in(0,1]$, then

$$
\mathbf{P}\{Y(t)>0\} \sim Q r(t)=\frac{\gamma}{c(1-\gamma)} r(t), \quad t \rightarrow \infty .
$$

Proof. From (5.1) and (5.3) we get that $\bar{F}(t) / Q, t \geq 0$ is a density on $[0, \infty)$

(a) Since $\theta>1$, then $r(t) / R$ is a density of a distribution on $[0, \infty)$. Now from Theorem 1, [2] we obtain that as $t \rightarrow \infty$,

$$
\begin{aligned}
& I(t)=\int_{0}^{t} r(t-u) \bar{F}(u) d u \\
= & Q R \int_{0}^{t} \frac{r(t-u)}{R} \frac{\bar{F}(u)}{Q} d u \sim Q R\left(\frac{r(t)}{R}+\frac{\bar{F}(t)}{Q}\right) \\
\sim & Q r(t)+R \bar{F}(t) .
\end{aligned}
$$


The assertion follows from the relation $1-e^{-x} \sim x, x \rightarrow 0$ and (5.4).

(b) Let $\Delta \in(0,1)$ be fixed. Then

$$
I(t)=\int_{0}^{t} \frac{\rho d u}{(t+1-u)^{\theta}(1+c u)^{1 / \gamma}}=\int_{0}^{t \Delta}+\int_{t \Delta}^{t}=I_{1}(t)+I_{2}(t) .
$$

For $I_{1}(t)$ we have

$$
I_{1}(t)=\int_{0}^{t \Delta} \frac{\rho d u}{(t+1-u)^{\theta}(1+c u)^{1 / \gamma}} \leq \frac{\rho}{(t(1-\Delta)+1)^{\theta}} \int_{0}^{t \Delta} \frac{d u}{(1+c u)^{1 / \gamma}},
$$

and

$$
I_{1}(t) \geq \frac{\rho}{(t+1)^{\theta}} \int_{0}^{t \Delta} \frac{d u}{(1+c u)^{1 / \gamma}} .
$$

Since, $\int_{0}^{t \Delta} \frac{d u}{(1+c u)^{1 / \gamma}} \rightarrow Q=\frac{\gamma}{c(1-\gamma)}, t \rightarrow \infty$ we obtain that

$$
1 \leq \liminf _{t \rightarrow \infty} \frac{I_{1}(t)}{Q r(t)} \leq \limsup _{t \rightarrow \infty} \frac{I_{1}(t)}{Q r(t)} \leq \frac{1}{1-\Delta} .
$$

For $I_{2}(t)$ will consider the cases $\theta \in(0,1)$ and $\theta=1$ separately.

If $\theta \in(0,1)$ then

$$
\begin{aligned}
& I_{2}(t)=\int_{t \Delta}^{t} \frac{\rho d u}{(t+1-u)^{\theta}(1+c u)^{1 / \gamma}} \leq \frac{\rho}{(1+c t \Delta)^{1 / \gamma}} \int_{0}^{t(1-\Delta)} \frac{d u}{(u+1)^{\theta}} \\
\leq & \frac{\rho}{(1+c t \Delta)^{1 / \gamma}} \times \frac{(t \Delta+1)^{1-\theta}}{1-\theta}=o(r(t)), t \rightarrow \infty,
\end{aligned}
$$

because of $1 / \gamma>1$.

Similarly, in the case when $\theta=1$ we have

$$
\begin{aligned}
& I_{2}(t)=\int_{t \Delta}^{t} \frac{\rho d u}{(t+1-u)(1+c u)^{1 / \gamma}} \leq \frac{\rho}{(1+c t \Delta)^{1 / \gamma}} \int_{0}^{t(1-\Delta)} \frac{d u}{(u+1)} \\
\leq & \frac{\rho}{(1+c t \Delta)^{1 / \gamma}} \times \log (t(1-\Delta)+1)=o(r(t)), t \rightarrow \infty,
\end{aligned}
$$

because of $1 / \gamma>1=\theta$ and $\log x$ varies slowly at infinity. Therefore, from (5.5), (5.6), and (5.7) we get

$$
1 \leq \liminf _{t \rightarrow \infty} \frac{I(t)}{Q r(t)} \leq \limsup _{t \rightarrow \infty} \frac{I(t)}{Q r(t)} \leq \frac{1}{1-\Delta} .
$$

Having in mind that $\Delta$ was arbitrary, we conclude that

$$
I(t) \sim Q r(t), \quad t \rightarrow \infty .
$$

Using again the relation $1-e^{-x} \sim x, \quad x \rightarrow 0$ and (5.4) we complete the proof of case (b). 
Theorem 3. Assume that (4.1) and (4.3) hold. Then:

$$
\mathbf{P}\{Y(t)>0\} \rightarrow 1-e^{-\rho Q}=1-e^{-\frac{\rho \gamma}{c(1-\gamma)}}, \quad t \rightarrow \infty .
$$

Proof. Following the same way as in the proof of case (b) in Theorem 2 we will prove that

$$
\lim _{t \rightarrow \infty} I(t)=\rho Q=\frac{\rho \gamma}{c(1-\gamma)} .
$$

Let $\Delta \in(0,1)$ be fixed. Then

$$
I(t)=\int_{0}^{t} \frac{r(t-u) d u}{(1+c u)^{1 / \gamma}}=\int_{0}^{t \Delta}+\int_{t \Delta}^{t}=I_{1}(t)+I_{2}(t) .
$$

For $I_{1}(t)$ we have

$$
I_{1}(t)=\int_{0}^{t \Delta} \frac{r(t-u) d u}{(1+c u)^{1 / \gamma}} \leq r(t(1-\Delta)) \int_{0}^{t \Delta} \frac{d u}{(1+c u)^{1 / \gamma}}
$$

and

$$
I_{1}(t) \geq r(t) \int_{0}^{t \Delta} \frac{d u}{(1+c u)^{1 / \gamma}}
$$

Since, $\int_{0}^{t \Delta} \frac{d u}{(1+c u)^{1 / \gamma}} \rightarrow Q, t \rightarrow \infty$, we obtain that

$$
\lim _{t \rightarrow \infty} I_{1}(t)=\rho Q .
$$

For $I_{2}(t)$ we have

$$
\begin{aligned}
& I_{2}(t)=\int_{t \Delta}^{t} \frac{r(t-u) d u}{(1+c u)^{1 / \gamma}} \leq \frac{\rho}{(1+c t \Delta)^{1 / \gamma}} \int_{0}^{t(1-\Delta)} d u \\
\leq & \frac{\rho t(1-\Delta)}{(1+c t \Delta)^{1 / \gamma}} \rightarrow 0, t \rightarrow \infty,
\end{aligned}
$$

because of $1 / \gamma>1$. Now (5.10), (5.11), and (5.9) prove (5.8) and the theorem.

Theorem 4. Assume that (4.1) and (4.4) hold. Then

$$
\mathbf{P}\{Y(t)>0\} \rightarrow 1, \quad t \rightarrow \infty .
$$

Proof. For $I(t)$ we have

$$
I(t)=\int_{0}^{t} \frac{\rho(t-u)^{\theta} d u}{(1+c u)^{1 / \gamma}} \geq \int_{0}^{t / 2} \frac{\rho(t-u)^{\theta} d u}{(1+c u)^{1 / \gamma}} \geq \rho(t / 2)^{\theta} \int_{0}^{t / 2} \frac{d u}{(1+c u)^{1 / \gamma}} .
$$

Since $\int_{0}^{t / 2} \frac{d u}{(1+c u)^{1 / \gamma}} \rightarrow \frac{\gamma}{c(1-\gamma)}, t \rightarrow \infty$, then $I(t) \rightarrow \infty, t \rightarrow \infty$. Now the assertion follows from the relation $e^{-x} \rightarrow 0, x \rightarrow \infty$ and (5.4). 


\section{Limit theorems}

In this section we will use also the following notations:

$$
\begin{aligned}
& Q(t ; s)=\int_{0}^{t} \bar{F}(u ; s) d u=\frac{\gamma(1-s)^{(1-\gamma)}}{c(1-\gamma)}\left(1-\left(1+c(1-s)^{\gamma} t\right)^{1-1 / \gamma}\right) \\
& Q(s)=\int_{0}^{\infty} \bar{F}(t ; s) d t=\int_{0}^{\infty} \frac{(1-s) d u}{\left(1+c u(1-s)^{\gamma}\right)^{1 / \gamma}}=\frac{\gamma(1-s)^{1-\gamma}}{c(1-\gamma)} .
\end{aligned}
$$

Let us consider the conditional p.g.f. of the process $Y(t), t \geq 0$,

$$
\mathbf{E}\left[s^{Y(t)} \mid Y(t)>0\right]=1-\frac{1-\Phi(t ; s)}{1-\Phi(t ; 0)}, \quad t \geq 0 .
$$

Depending on the variability of the rate $r(t)$ we obtain different types limit theorems.

Theorem 5. Assume that (4.1) and (4.2) with $\theta>1$ hold true.

(a) If $\bar{F}(t)=o(r(t)), \quad t \rightarrow \infty$, then

$$
\lim _{t \rightarrow \infty} \mathbf{P}\{Y(t)=k \mid Y(t)>0\}=p_{k}, k=1,2, \ldots,
$$

where

$$
\sum_{k=1}^{\infty} p_{k} s^{k}=1-(1-s)^{1-\gamma}, \quad s \in[0,1] .
$$

(b) If $r(t)=o(\bar{F}(t)), \quad t \rightarrow \infty$, then

$$
\lim _{t \rightarrow \infty} \mathbf{P}\{Y(t) \bar{F}(t) \leq x \mid Y(t)>0\}=D_{\gamma}(x), \quad x \geq 0,
$$

where $D_{\gamma}(x)$ has Laplace transform $\hat{D}_{\gamma}(\lambda)=1-\lambda\left(1+\lambda^{\gamma}\right)^{-1 / \gamma}, \quad \lambda>0$.

(c) If $r(t) / \bar{F}(t) \rightarrow d \in(0, \infty)$, then

$$
\begin{aligned}
& \lim _{t \rightarrow \infty} \mathbf{P}\{Y(t)=k \mid Y(t)>0\}=p_{k}, k=1,2, \ldots, \\
& \sum_{k=1}^{\infty} p_{k} s^{k}=\frac{Q}{Q+R / d}\left(1-(1-s)^{1-\gamma}\right), \quad s \in[0,1],
\end{aligned}
$$

and

$$
\lim _{t \rightarrow \infty} \mathbf{P}\{Y(t) \bar{F}(t) \leq x \mid Y(t)>0\}=\frac{Q d}{R+Q d}+\frac{R}{R+Q d} D_{\gamma}(x), \quad x \geq 0 .
$$

Proof. Under the conditions of the theorem we have that for any fixed $s \in[0,1) \bar{F}(t, s) / Q(s)$ is a density on $[0, \infty)$. On the other hand, we have also that $r(t) / R$ is a density on $[0, \infty)$. Therefore, as in the proof of Theorem 2(a) we get by Theorem 1, [2] that for any fixed $s \in[0,1)$,

$$
I(t ; s)=\int_{0}^{t} r(t-u) \bar{F}(t ; s) d u \sim Q(s) r(t)+\bar{F}(t ; s) R, \quad t \rightarrow \infty .
$$


By the relation $1-e^{-x} \sim x, \quad x \rightarrow 0$, we get that for any fixed $s \in[0,1)$

$$
1-\Phi(t ; s)=1-\exp (-I(t ; s)) \sim Q(s) r(t)+\bar{F}(t ; s) R, \quad t \rightarrow \infty .
$$

(a) In this case $\bar{F}(t)=o(r(t))$ as $t \rightarrow \infty$. Using the relation (5.5) we get

$$
\frac{1-\Phi(t ; s)}{1-\Phi(t ; 0)} \sim \frac{Q(s) r(t)+\bar{F}(t ; s) R}{Q r(t)+\bar{F}(t) R}=\frac{Q(s)+\frac{\bar{F}(t ; s)}{r(t)} R}{Q+\frac{\bar{F}(t)}{r(t)} R} .
$$

By the assumption in this case we have that

$$
\frac{\bar{F}(t)}{r(t)} \rightarrow 0, \quad \frac{\bar{F}(t ; s)}{r(t)} \leq \frac{\bar{F}(t)}{r(t)} \rightarrow 0, \quad t \rightarrow \infty
$$

for any fixed $s \in[0,1)$. Therefore, we get

$$
\frac{1-\Phi(t ; s)}{1-\Phi(t ; 0)} \rightarrow \frac{Q(s)}{Q}=(1-s)^{1-\gamma}, \quad t \rightarrow \infty .
$$

Using equation (6.3) we complete the proof.

(b) Let us assume that $r(t)=o(\bar{F}(t)), \quad t \rightarrow \infty$.

In this case the whole mass goes to infinity. Now we will consider the behavior of the sample paths under an appropriate normalization, which provides a proper continuous limiting distribution. Let us denote by $s(t)=\exp (-\lambda \bar{F}(t))=$ $\exp \left(-\lambda(1+c t)^{-1 / \gamma}\right)$. For an arbitrary but fixed $\Delta \in(0,1)$ we have

$$
I(t ; s(t))=\int_{0}^{t} \bar{F}(t-u ; s(t)) r(u) d u=\int_{0}^{t \Delta}+\int_{t \Delta}^{t}=I_{1}(t ; s(t))+I_{2}(t ; s(t))
$$

For $I_{1}(t ; s(t))$ we have

$$
\begin{gathered}
I_{1}(t ; s(t))=\int_{0}^{t \Delta} \frac{(1-s(t)) r(u) d u}{\left(1+c(t-u)(1-s(t))^{\gamma}\right)^{1 / \gamma}} \\
\leq \frac{1-s(t)}{\left(1+c t(1-\Delta)(1-s(t))^{\gamma}\right)^{1 / \gamma}} \int_{0}^{t \Delta} r(u) d u \\
\\
I_{1}(t ; s(t)) \geq \frac{1-s(t)}{\left(1+c t(1-s(t))^{\gamma}\right)^{1 / \gamma}} \int_{0}^{t \Delta} r(u) d u .
\end{gathered}
$$

Using the well known asymptotic $1-e^{-x} \sim x, \quad x \rightarrow 0$ we have that

$$
1-s(t)=1-\exp (-\lambda \bar{F}(t)) \sim \lambda \bar{F}(t), \quad t \rightarrow \infty .
$$

This relation provides that

$$
\begin{aligned}
& \limsup _{t \rightarrow \infty} \frac{I_{1}(t ; s(t))}{\bar{F}(t)} \leq \limsup _{t \rightarrow \infty} \frac{1-s(t)}{\bar{F}(t)} \frac{1}{\left(1+c t(1-\Delta)(1-s(t))^{\gamma}\right)^{1 / \gamma}} \int_{0}^{t \Delta} r(u) d u \\
& =\frac{R \lambda}{\left(1+(1-\Delta) \lambda^{\gamma}\right)^{1 / \gamma}}
\end{aligned}
$$


and

$$
\begin{aligned}
& \liminf _{t \rightarrow \infty} \frac{I_{1}(t ; s(t))}{\bar{F}(t)} \geq \liminf _{t \rightarrow \infty} \frac{1-s(t)}{\bar{F}(t)} \frac{1}{\left(1+c t(1-s(t))^{\gamma}\right)^{1 / \gamma}} \int_{0}^{t \Delta} r(u) d u \\
= & \frac{R \lambda}{\left(1+\lambda^{\gamma}\right)^{1 / \gamma}} .
\end{aligned}
$$

On the other hand for $I_{2}(t ; s(t))$ we obtain

$$
\begin{aligned}
& I_{2}(t ; s(t))=\int_{t \Delta}^{t} \frac{(1-s(t)) r(u) d u}{\left(1+c(t-u)(1-s(t))^{\gamma}\right)^{1 / \gamma}} \\
\leq & (1-s(t)) r(t \Delta) \int_{0}^{t(1-\Delta)} \frac{d u}{\left(1+c u(1-s(t))^{\gamma}\right)^{1 / \gamma}} \\
\leq & \frac{(1-s(t)) r(t \Delta)}{c(1-s(t))^{\gamma}} \int_{0}^{t(1-\Delta)} \frac{d\left(1+c u(1-s(t))^{\gamma}\right)}{\left(1+c u(1-s(t))^{\gamma}\right)^{1 / \gamma}} \\
\leq & \frac{(1-s(t))^{1-\gamma} r(t \Delta)}{c}\left[\frac{\left(1+c t(1-\Delta)(1-s(t))^{\gamma}\right)^{1-1 / \gamma}}{1-1 / \gamma}-\frac{1}{1-1 / \gamma}\right] .
\end{aligned}
$$

From here it is not difficult to derive that

$$
\lim _{t \rightarrow \infty} \frac{I_{2}(t ; s(t))}{\bar{F}(t)}=0 .
$$

Now from the relations for $I_{1}(t ; s(t))$ and $I_{2}(t ; s(t))$ we obtain that

$$
\frac{R \lambda}{\left(1+\lambda^{\gamma}\right)^{1 / \gamma}} \leq \liminf _{t \rightarrow \infty} \frac{I(t ; s(t))}{\bar{F}(t)} \leq \limsup _{t \rightarrow \infty} \frac{I(t ; s(t))}{\bar{F}(t)} \leq \frac{R \lambda}{\left(1+(1-\Delta) \lambda^{\gamma}\right)^{1 / \gamma}} .
$$

Since $\Delta$ was arbitrary we conclude that

which gives that

$$
I(t ; s(t)) \sim \bar{F}(t) \frac{R \lambda}{\left(1+\lambda^{\gamma}\right)^{1 / \gamma}},
$$

$$
1-\Phi(t ; s(t)) \sim \bar{F}(t) \frac{R \lambda}{\left(1+\lambda^{\gamma}\right)^{1 / \gamma}},
$$

using the asymptotic $1-e^{-x} \sim x, \quad x \rightarrow 0$. In this case we have from (5.5) that

$$
1-\Phi(t ; 0) \sim \bar{F}(t) R, \quad t \rightarrow \infty .
$$

Therefore

$$
\lim _{t \rightarrow \infty} \mathbf{E}\left[e^{-\lambda Y(t) \bar{F}(t)} \mid Y(t)>0\right]=\hat{D}_{\gamma}(\lambda)=1-\frac{\lambda}{\left(1+\lambda^{\gamma}\right)^{1 / \gamma}}, \quad \lambda>0,
$$

which completes the proof in this case.

(c) The proof of this case follows the proof of case (a) for trajectories without normalization and the proof of case (b) for the trajectories that go to infinity. 
Theorem 6. Assume that (4.1) and (4.2) with $\theta \in(0,1]$ hold. Then

$$
\begin{aligned}
& \lim _{t \rightarrow \infty} \mathbf{P}\{Y(t)=k \mid Y(t)>0\}=p_{k}, k=1,2, \ldots, \\
& \sum_{k=1}^{\infty} p_{k} s^{k}=1-(1-s)^{1-\gamma}, s \in[0,1] .
\end{aligned}
$$

Pr o of. Let $\Delta \in(0,1)$ be fixed. Then

$$
\begin{aligned}
I(t ; s) & =\int_{0}^{t} r(t-u) \frac{1-s}{\left(1+c u(1-s)^{\gamma}\right)^{1 / \gamma}} d u \\
& =\int_{0}^{t \Delta}+\int_{t \Delta}^{t}=I_{1}(t ; s)+I_{2}(t ; s) .
\end{aligned}
$$

For $I_{1}(t ; s)$ we have

and

$$
I_{1}(t ; s) \leq r(t(1-\Delta)) \int_{0}^{t \Delta} \frac{(1-s) d u}{\left(1+c u(1-s)^{\gamma}\right)^{1 / \gamma}}
$$

Since

$$
I_{1}(t ; s) \geq r(t) \int_{0}^{t \Delta} \frac{(1-s) d u}{\left(1+c u(1-s)^{\gamma}\right)^{1 / \gamma}}
$$

we obtain that

$$
\int_{0}^{t \Delta} \frac{(1-s) d u}{\left(1+c u(1-s)^{\gamma}\right)^{1 / \gamma}} \rightarrow Q(s)=\frac{\gamma(1-s)^{1-\gamma}}{c(1-\gamma)}, t \rightarrow \infty
$$

$$
1 \leq \liminf _{t \rightarrow \infty} \frac{I_{1}(t ; s)}{Q(s) r(t)} \leq \limsup _{t \rightarrow \infty} \frac{I_{1}(t ; s)}{Q(s) r(t)} \leq \frac{1}{1-\Delta} .
$$

Since $\bar{F}(t ; s) \leq \bar{F}(t)$ we have that

$$
I_{2}(t ; s)=\int_{t \Delta}^{t} r(t-u) \bar{F}(u ; s) d u \leq \int_{t \Delta}^{t} r(t-u) \bar{F}(u) d u .
$$

From (5.6), and (5.7) we obtain that $I_{2}(t ; s)=o(r(t)), \quad t \rightarrow \infty$. This relation, (6.5), and (6.4) yield that

$$
1 \leq \liminf _{t \rightarrow \infty} \frac{I(t ; s)}{Q(s) r(t)} \leq \limsup _{t \rightarrow \infty} \frac{I(t ; s)}{Q(s) r(t)} \leq \frac{1}{1-\Delta} .
$$

Having in mind that $\Delta$ was arbitrary, we conclude that

$$
I(t ; s) \sim Q(s) r(t), \quad t \rightarrow \infty .
$$

Using the relation $1-e^{-x} \sim x, \quad x \rightarrow 0$, we have that

$$
1-\Phi(t ; s) \sim Q(s) r(t), \quad t \rightarrow \infty .
$$

Now the proof follows from Theorem 2, (ii) and (6.3). 
Theorem 7. Assume that (4.1) and (4.3) hold. Then

$$
\begin{aligned}
& \lim _{t \rightarrow \infty} \mathbf{P}\{Y(t)=k \mid Y(t)>0\}=p_{k}, k=1,2, \ldots, \\
& \sum_{k=1}^{\infty} p_{k} s^{k}=\exp (-\rho Q(s))=\exp \left(-\rho \frac{\gamma(1-s)^{1-\gamma}}{c(1-\gamma)}\right), s \in[0,1] .
\end{aligned}
$$

Proof. We have

$$
I(t ; s)=\int_{0}^{t} r(t-u) \bar{F}(u ; s) d u=\int_{0}^{t / 2}+\int_{t / 2}^{t}=I_{1}(t ; s)+I_{2}(t ; s) .
$$

Let $\varepsilon>0$ be fixed. For every $t$ large enough we have

$$
\rho(1-\varepsilon) \int_{0}^{t / 2} \bar{F}(u ; s) d u \leq I_{1}(t ; s) \leq \rho(1+\varepsilon) \int_{0}^{t / 2} \bar{F}(u ; s) d u .
$$

Since

$$
\int_{0}^{t \Delta} \bar{F}(u ; s) d u \rightarrow Q(s), t \rightarrow \infty
$$

we obtain that

$$
(1-\varepsilon) \rho Q(s) \leq \liminf _{t \rightarrow \infty} I_{1}(t ; s) \leq \limsup _{t \rightarrow \infty} I_{1}(t ; s) \leq(1+\varepsilon) \rho Q(s) .
$$

Since $\bar{F}(t ; s) \leq \bar{F}(t)$ we have that

$$
I_{2}(t ; s)=\int_{t / 2}^{t} r(t-u) \bar{F}(u ; s) d u \leq F(t / 2) \int_{0}^{t / 2} r(u) d u \rightarrow 0, t \rightarrow \infty .
$$

This relation, (6.7), and (6.6) yield that

$$
(1-\varepsilon) \rho Q(s) \leq \liminf _{t \rightarrow \infty} I(t ; s) \leq \limsup _{t \rightarrow \infty} I(t ; s) \leq(1+\varepsilon) \rho Q(s) .
$$

Having in mind that $\varepsilon>0$ was arbitrary, we complete the proof of the theorem.

Theorem 8. Assume that (4.1) and (4.4) hold.

(a) If $0<\gamma<\frac{1}{1+\theta}$, then

$$
\lim _{t \rightarrow \infty} \mathbf{E}\left[\exp \left(-\lambda Y(t) t^{-\theta /(1-\gamma)}\right)\right]=\exp \left(-\frac{\rho \gamma}{c(1-\gamma)} \lambda^{1-\gamma}\right), \quad \lambda>0
$$

which is the Laplace transform of a stable distribution $D(x)$ with exponent $1-\gamma$, and

$$
1-D(x) \sim \frac{\rho \gamma}{c \Gamma(2-\gamma)} x^{-(1-\gamma)} \quad x \rightarrow \infty
$$


(b) If $\gamma=\frac{1}{1+\theta}$, then

$$
\begin{aligned}
& \lim _{t \rightarrow \infty} \mathbf{E}\left[\exp \left(-\lambda Y(t) t^{-(\theta+1)}\right)\right] \\
= & \hat{D}(\lambda):=\exp \left(-c^{-1 / \gamma} \int_{0}^{c \lambda^{\gamma} /\left(1+c \lambda^{\gamma}\right)} u^{(1-\gamma) / \gamma}(1-u)^{-1 / \gamma} d u\right), \quad \lambda>0,
\end{aligned}
$$

which by the continuity theorem for Laplace transform provides that

$$
\lim _{t \rightarrow \infty} \mathbf{P}\left\{Y(t) t^{-(1+\theta)} \leq x\right\}=D(x), x \geq 0,
$$

where $D(x)$ has Laplace transform $\hat{D}(\lambda)$.

(c) If $\frac{1}{1+\theta}<\gamma<1$, then

$$
\lim _{t \rightarrow \infty} \Phi\left(t ; \lambda t^{-(1+\theta)}\right)=\exp \left(-\frac{\rho \lambda}{1+\theta}\right), \quad \lambda>0,
$$

which is the Laplace transform of a degenerate distribution with mass 1 at the point $\rho /(\theta+1)$, that is

$$
\lim _{t \rightarrow \infty} \mathbf{P}\left\{Y(t) t^{-(1+\theta)} \leq x\right\}= \begin{cases}0, & x<\frac{\rho}{1+\theta} \\ 1, & x \geq \frac{\rho}{1+\theta}\end{cases}
$$

Proof. (a) We set $s(t)=e^{-\lambda t^{-\theta /(1-\gamma)}}$. For $\Delta \in(0,1)$ we have

$$
I(t ; s(t))=\int_{0}^{t} \frac{\rho(1-s(t))(t-u)^{\theta} d u}{\left(1+c u(1-s(t))^{\gamma}\right)^{1 / \gamma}}=\int_{0}^{t \Delta}+\int_{t \Delta}^{t}=I_{1}(t ; s(t))+I_{2}(t ; s(t)) .
$$

For $I_{1}(t ; s(t))$ we obtain

$$
\begin{aligned}
& I_{1}(t ; s(t)) \leq \frac{\rho(1-s(t)) t^{\theta}}{c(1-s(t))^{\gamma}} \int_{0}^{t \Delta} \frac{d\left(1+c u(1-s(t))^{\gamma}\right)}{\left(1+c u(1-s(t))^{\gamma}\right)^{1 / \gamma}} \\
= & \frac{\rho \gamma(1-s(t))^{1-\gamma} t^{\theta}}{c(1-\gamma)}\left(1-\left(1+c t \Delta(1-s(t))^{\gamma}\right)^{1-1 / \gamma}\right) .
\end{aligned}
$$

Since $s(t)=e^{-\lambda t^{-\theta /(1-\gamma)}}$, using the relation $1-e^{-\lambda / t^{-\theta /(1-\gamma)}}=\lambda t^{-\theta /(1-\gamma)}(1+$ $o(1)), \quad t \rightarrow \infty$ we obtain that as $t \rightarrow \infty$,

$$
\begin{aligned}
& (1-s(t))^{1-\gamma} \rightarrow 0, t^{\theta}(1-s(t))^{1-\gamma} \rightarrow \lambda^{1-\gamma}, \\
& t(1-s(t))^{\gamma} \sim \lambda^{\gamma} t^{1-(\theta \gamma) /(1-\gamma)}, \quad\left(1+c t(1-s(t))^{\gamma}\right)^{1-1 / \gamma} \rightarrow 0 .
\end{aligned}
$$

Therefore

$$
\lim _{t \rightarrow \infty}\left(\rho \gamma \frac{t^{\theta}(1-s(t))^{1-\gamma}}{c(1-\gamma)}\right)\left(1-\left(1+c t \Delta(1-s(t))^{\gamma}\right)^{1-1 / \gamma}\right)=\frac{\rho \gamma}{c(1-\gamma)} \lambda^{1-\gamma} .
$$


In the same way we obtain that as $t \rightarrow \infty$,

$$
\begin{aligned}
& I_{1}(t ; s(t)) \geq \frac{\rho \gamma(1-s(t))^{1-\gamma} t^{\theta}(1-\Delta)^{\theta}}{c(1-\gamma)}\left(1-\left(1+c t \Delta(1-s(t))^{\gamma}\right)^{1-1 / \gamma}\right) \\
\rightarrow & (1-\Delta)^{\theta} \frac{\rho \gamma}{c(1-\gamma)} \lambda^{1-\gamma} .
\end{aligned}
$$

On the other hand for $I_{2}(t, s(t))$ we have

$$
\begin{aligned}
& 0 \leq I_{2}(t ; s(t)) \leq \frac{1-s(t)}{\left(c t \Delta(1-s(t))^{\gamma}\right)^{1 / \gamma}} \int_{0}^{t(1-\Delta)} \rho u^{\theta} d u=\rho(c \Delta)^{-1 / \gamma} t^{-1 / \gamma} \frac{(t \Delta)^{\theta+1}}{\theta+1} \\
= & \rho \frac{\Delta^{-1 / \gamma+\theta+1} t^{-1 / \gamma+\theta+1}}{c^{1 / \gamma}(\theta+1)} \rightarrow 0, \quad t \rightarrow \infty,
\end{aligned}
$$

since $-1 / \gamma+1+\theta<0$. Therefore

$$
(1-\Delta)^{\theta} \frac{\rho \gamma}{c(1-\gamma)} \lambda^{1-\gamma} \leq \liminf _{t \rightarrow \infty} I(t ; s(t)) \leq \limsup _{t \rightarrow \infty} I(t ; s(t)) \leq \frac{\rho \gamma}{c(1-\gamma)} \lambda^{1-\gamma}
$$

Since $\Delta \in(0,1)$ was arbitrary we obtain that

Therefore

$$
\lim _{t \rightarrow \infty} I(t ; s(t))=\frac{\rho \gamma}{c(1-\gamma)} \lambda^{1-\gamma}
$$

$$
\lim _{t \rightarrow \infty} \Phi(t ; s(t))=\exp \left(-\frac{\rho \gamma}{c(1-\gamma)} \lambda^{1-\gamma}\right), \quad \lambda>0,
$$

which is the Laplace transform of a stable distribution $D(x)$ with exponent $1-\gamma$, (see [14], p. $146(5.4 .3),(5.4 .6))$,

$$
1-D(x) \sim \frac{\rho \gamma}{c \Gamma(2-\gamma)} x^{-(1-\gamma)} \quad x \rightarrow \infty
$$

(b) Now $\gamma=\frac{1}{1+\theta}$. Then we set $s(t)=e^{-\lambda t^{-(1+\theta)}}$. Let $\varepsilon>0$ be fixed. For every $t$ large enough we have

$$
(1-\varepsilon) \lambda t^{-(\theta+1)} \leq 1-s(t) \leq(1+\varepsilon) \lambda t^{-(\theta+1)} .
$$

Using these inequalities we obtain for

$$
I(t ; s(t))=\int_{0}^{t} \frac{(1-s(t)) \rho(t-u)^{\theta} d u}{\left(1+c u(1-s(t))^{\gamma}\right)^{1 / \gamma}}
$$

that

$$
\begin{aligned}
& (1-\varepsilon) \rho \lambda \int_{0}^{t} \frac{t^{-(\theta+1)}(t-u)^{\theta} d u}{\left(1+c(1+\varepsilon) \lambda^{1 /(\theta+1)} u t^{-1}\right)^{\theta+1}} \leq I(t ; s(t)) \\
\leq & (1+\varepsilon) \rho \lambda \int_{0}^{t} \frac{t^{-(\theta+1)}(t-u)^{\theta} d u}{\left(1+c(1-\varepsilon) \lambda^{1 /(\theta+1)} u t^{-1}\right)^{\theta+1}} .
\end{aligned}
$$


Substitute $u=v t$ in the above integrals to get

$$
\begin{aligned}
& (1 \pm \varepsilon) \rho \lambda \int_{0}^{t} \frac{t^{-(\theta+1)}(t-u)^{\theta} d u}{\left(1+c(1 \mp \varepsilon) \lambda^{1 /(\theta+1)} u t^{-1}\right)^{\theta+1}} \\
= & (1 \pm \varepsilon) \rho \lambda \int_{0}^{1} \frac{(1-v)^{\theta} d v}{\left(1+c(1 \mp \varepsilon) \lambda^{1 /(\theta+1)} v\right)^{\theta+1}} .
\end{aligned}
$$

Since $\varepsilon>0$ was arbitrary it is not difficult to conclude that

$$
\begin{aligned}
& \lim _{t \rightarrow \infty} I(t ; s(t))=\rho \lambda \int_{0}^{1} \frac{(1-v)^{\theta} d v}{\left(1+c \lambda^{1 /(\theta+1)} v\right)^{\theta+1}} \\
= & \rho c^{-(\theta+1)} \int_{0}^{1} \frac{(1-v)^{\theta} d v}{\left(\frac{1}{c \lambda^{1 /(\theta+1)}}+v\right)^{\theta+1}}=c^{-(\theta+1)} \int_{0}^{1} \frac{(1-v)^{\theta} d v}{(K+v)^{\theta+1}}
\end{aligned}
$$

where we denote $K=\frac{1}{c \lambda^{1 /(\theta+1)}}$. Changing variables $v=1-u(1+K)$ we finally get

$$
\begin{gathered}
\lim _{t \rightarrow \infty} I(t ; s)=-\rho c^{-(\theta+1)} \int_{1-K /(1+K)}^{0} \frac{u^{\theta} d u}{(1-u)^{1+\theta}} \\
=\rho c^{-1 / \gamma} \int_{0}^{c \lambda^{\gamma} /\left(1+c \lambda^{\gamma}\right)} u^{(1-\gamma) / \gamma}(1-u)^{-1 / \gamma} d u .
\end{gathered}
$$

Therefore

$$
\begin{aligned}
& \lim _{t \rightarrow \infty} \Phi\left(t ; \exp \left(-\lambda t^{-(\theta+1)}\right)\right) \\
= & \hat{D}(\lambda):=\exp \left(-\rho c^{-1 / \gamma} \int_{0}^{c \lambda^{\gamma} /\left(1+c \lambda^{\gamma}\right)} u^{(1-\gamma) / \gamma}(1-u)^{-1 / \gamma} d u\right), \quad \lambda>0,
\end{aligned}
$$

which by the continuity theorem for Laplace transform, provides that

$$
\lim _{t \rightarrow \infty} \mathbf{P}\left\{Y(t) t^{-1 / \gamma} \leq x\right\}=D(x), x \geq 0,
$$

where $D(x)$ has Laplace transform $\hat{D}(\lambda)$.

(c) Let now $\frac{1}{1+\theta}<\gamma<1, \theta>0, r(t)=\rho t^{\theta}$. In this case we set $s(t)=$ $\exp \left(-\lambda t^{-(\theta+1)}\right), \quad \lambda>0$.

For an arbitrary but fixed $\Delta \in(0,1)$ we have as in the case (a) that

$$
I(t ; s(t))=\int_{0}^{t} \frac{\rho(1-s(t))(t-u)^{\theta} d u}{\left(1+c u(1-s(t))^{\gamma}\right)^{1 / \gamma}}=\int_{0}^{t \Delta}+\int_{t \Delta}^{t}=I_{1}(t ; s(t))+I_{2}(t ; s(t)) .
$$

For $I_{1}(t ; s)$ we obtain

$$
I_{1}(t ; s(t)) \leq \frac{\rho \gamma(1-s(t))^{1-\gamma} t^{\theta}}{c(1-\gamma)}\left(1-\left(1+c t \Delta(1-s(t))^{\gamma}\right)^{1-1 / \gamma}\right) .
$$


Using the relation $1-e^{-\lambda / t^{-(1+\theta)}}=\lambda t^{-(1+\theta)}(1+o(1))$, we obtain that

$$
(1-s(t)) t^{1+\theta} \rightarrow \lambda, t(1-s(t))^{\gamma} \sim \lambda^{\gamma} t^{1-(\theta \gamma) /(1-\gamma)} \rightarrow 0,
$$

as $t \rightarrow \infty$. Then from the relation $1-(1+x)^{k} \sim-k x, x \rightarrow 0$ we have that

$$
1-\left(1+c t \Delta(1-s(t))^{\gamma}\right)^{1-1 / \gamma} \sim-\left(1-\frac{1}{\gamma}\right) c t \Delta(1-s(t))^{\gamma}, t \rightarrow \infty .
$$

Therefore

$$
\begin{aligned}
& 0 \leq I_{1}(t ; s(t)) \leq \frac{\rho \gamma(1-s(t))^{1-\gamma} t^{\theta}}{c(1-\gamma)}\left(1-\left(1+c t \Delta(1-s(t))^{\gamma}\right)^{1-1 / \gamma}\right) \\
\sim & -\frac{\rho \gamma(1-s(t))^{1-\gamma} t^{\theta}}{c(1-\gamma)}\left(1-\frac{1}{\gamma}\right) c t \Delta(1-s(t))^{\gamma} \rightarrow \Delta \lambda, \quad t \rightarrow \infty .
\end{aligned}
$$

On the other hand we have for $I_{2}(t ; s)$ that

$$
\begin{aligned}
& I_{2}(t ; s(t)) \leq \frac{\rho(1-s(t))}{\left(1+c t \Delta(1-s(t))^{\gamma}\right)^{1 / \gamma}} \int_{0}^{t(1-\Delta)} u^{\theta} d u \\
= & \frac{\rho(1-s(t))}{\left(1+c t \Delta(1-s(t))^{\gamma}\right)^{1 / \gamma}} \frac{t^{\theta+1}(1-\Delta)^{\theta+1}}{\theta+1} \\
= & \frac{(1-\Delta)^{\theta+1}}{\theta+1} \frac{\rho(1-s(t)) t^{\theta+1}}{\left(1+c t \Delta(1-s(t))^{\gamma}\right)^{1 / \gamma}} \rightarrow \frac{(1-\Delta)^{\theta+1}}{\theta+1} \rho \lambda, \quad t \rightarrow \infty .
\end{aligned}
$$

Similarly we obtain for $I_{2}(t ; s)$ that as $t \rightarrow \infty$,

$$
\begin{aligned}
& I_{2}(t ; s(t)) \geq \frac{\rho(1-s(t))}{\left(1+c t(1-s(t))^{\gamma}\right)^{1 / \gamma}} \int_{0}^{t(1-\Delta)} u^{\theta} d u \\
= & \frac{(1-\Delta)^{\theta+1}}{\theta+1} \frac{\rho(1-s(t)) t^{\theta+1}}{\left(1+c t(1-s(t))^{\gamma}\right)^{1 / \gamma}} \rightarrow \frac{(1-\Delta)^{\theta+1}}{\theta+1} \rho \lambda .
\end{aligned}
$$

Therefore

$$
\frac{(1-\Delta)^{\theta+1}}{\theta+1} \rho \lambda \leq \liminf _{t \rightarrow \infty} I(t ; s(t)) \leq \limsup _{t \rightarrow \infty} I(t ; s(t)) \leq \Delta \rho \lambda+\frac{(1-\Delta)^{\theta+1}}{\theta+1} \rho \lambda .
$$

Since $\Delta \in(0,1)$ was arbitrary we obtain that $\lim _{t \rightarrow \infty} I(t ; s)=\frac{\rho \lambda}{1+\theta}, \quad \lambda>0$. Therefore

$$
\lim _{t \rightarrow \infty} \Phi(t ; s(t))=\exp \left(-\frac{\rho \lambda}{1+\theta}\right), \lambda>0,
$$

which is the Laplace transform of a degenerate distribution with mass 1 at the point $\rho /(\theta+1)$. This completes the proof of the case (c) and the theorem. 
Remark 1. Since the limit is degenerate at one point we have also the convergence in probability,

$$
Y(t) t^{-(1+\theta)} \rightarrow \frac{\rho}{1+\theta}, \quad t \rightarrow \infty,
$$

which can be interpreted as a LLN.

Acknowledgment. The research was partially supported by the National Fund for Scientific Research at the Ministry of Education and Science of Bulgaria, grant No. DFNI-I02/17.

\section{REFERENCES}

[1] K. B. Athreya, P. E. Ney. Branching Processes. New York, Springer, 1972.

[2] N. H. Bingham, C. M. Goldie, E. Omey. Regularly varying probability densities. Publications de l'Institut Mathématique (Beograd), Novel serie, 80(94) (2006), 47-57.

[3] J. A. Foster. A limit theorem for a branching process with state dependent immigration. Ann. Math. Stat., 42, (1971), 1773-1776.

[4] C. R. Heathcote. A branching process allowing immigration. J. Roy. Statist. Soc., Ser. B, 27, No 1 (1965), 138-143.

[5] O. Hyrien, K. V. Mitov, N. M. Yanev. Limit theorems for supercritical Markov branching processes with non-homogeneous Poisson immigration. C. R. Acad. Bulg. Sci., 66, No 4, (2013), 485-493.

[6] O. Hyrien, K. V. Mitov, N. M. Yanev. Limit theorems for subcritical Markov branching processes with non-homogeneous Poisson immigration. C. R. Acad. Bulg. Sci., 68, No 3 (2015), 313-320.

[7] K. V. Mitov, N. M. YAnev. Sevastyanov branching processes with nonhomogeneous Poisson immigration. Proc. the Steklov Inst. Math., 282, (2013), 172-185.

[8] A. G. Pakes. A branching process with a state dependent immigration component. Adv. Appl. Probab., 3, (1971), 301-314.

[9] A. G. PAKEs. Critical Markov branching processes limit theorems allowing infinite variance. Adv. Appl. Probab., 42, No 2 (2010), 460-488.

[10] B. A. Sevastyanov. Branching processes. Moscow, Nauka, 1971 (in Russian). 
[11] B. A. Sevastyanov. Limit theorems for branching stochastic processes of special form. Teor. Veroyatn. Primen., 2 (1957), 339-348 (in Russian).

[12] S. Sagitov, A. Lindo. A special family of Galton-Watson processes with explosions. In: Branching Processes and Their Applications. Proceedings of the workshop, WBPA, Badajoz, Spain, April 7-10, 2015. (Eds I.M. del Puerto et al.) Lecture Notes in Statistics, vol. 219 2016, 237-254.

[13] R. S. SLACK A branching process with mean one and possibly infinite variance. Z. Wahrscheinlichkeitstheor. Verw. Geb., 9 (1968), 139-145.

[14] V. V. Uchaikin, V. M. Zolotarev. Chance and Stability. Stable distributions and their Applications. Modern Probability and Statistics. Utrecht, VSP, 1999.

[15] V. A. Vatutin. Branching processes with infinite variance. In: Proc. 4th International Summer School on Probability Theory and Math. Statistics, Varna, Bulgaria, October 25-November 7, 1982. Sofia, Publishing House of the Bulgarian Academy of Sciences, 1983, 9-38.

[16] A. M. Yaglom. Some limit theorems in the theory of stochastic branching processes. Dokl. Akad. Nauk SSSR, 56, No 8, (1947), 795-798.

[17] A. M. Yakovlev, N. M. YAnev. Branching stochastic processes with immigration in analysis of renewing cell populations. Math. Biosci., 203, No 1 (2006), 37-63.

[18] V. M. Zolotarev. More exact statement of several limit theorems in the theory of branching processes, Teoriya Veroyatnostei $i$ ee Primeneniya, 2, No 2 (1957), 256-266 (in Russian); English translation in: Theor. Prob. Appl., 2, No 2 (1958), 245-253.

Kosto V. Mitov

Faculty of Aviation

National Military University "Vasil Levski"

5856 D. Mitropolia, Pleven, Bulgaria

E-mail: kmitov@yahoo.com

Nikolay M. Yanev

Department of Probability and Statistics

Institute of Mathematics and Informatics

Bulgarian Academy of Sciences,

1113 Sofia, Bulgaria

E-mail: yanev@math.bas.bg 